\title{
Micropatterned Structures for Studying the Mechanics of Biological Polymers
}

\author{
Henry T. Schek, III and Alan J. Hunt* \\ Department of Biomedical Engineering, University of Michigan, \\ Ann Arbor, MI, USA \\ E-mail: tschek@umich.edu
}

\begin{abstract}
Studying the mechanics of nanometer-scale biomolecules presents many challenges; these include maintaining light microscopy image quality and avoiding interference with the laser used for mechanical manipulation, that is, optical tweezers. Studying the pushing forces of a polymerizing filament requires barriers that meet these requirements and that can impede and restrain nanoscale structures subject to rapid thermal movements. We present a flexible technique that meets these criteria, allowing complex barrier geometries with undercut sidewall profiles to be produced on \#1 cover glass for the purpose of obstructing and constraining polymerizing filaments, particularly microtubules. Using a two-layer lithographic process we are able to separate the construction of the primary features from the construction of a depth and shape-controlled undercut. The process can also be extended to create a large uniform gap between an SU-8 photoresist layer and the glass substrate. This technique can be easily scaled to produce large quantities of shelfstable, reusable microstructures that are generally applicable to microscale studies of the interaction of cellular structures with defined microscale features.
\end{abstract}

Key Words. microtubule, optical tweezers, biopolymer, SU-8

\section{Introduction}

Optical tweezers have become a core tool for studying force and displacement generated by single biological molecules (e.g. Allersma et al., 1998; Baldi et al., 2003; Felgner et al., 1996; Mehta et al., 1998; Meiners and Quake, 2000; Visscher et al., 1999). Typically, optical tweezers are applied to manipulate and measure forces on a trapped microbead attached to a molecule of interest. Optical tweezers have generally been used to study molecules that pull or tug against the resistance of the optical trap; however, biological structures are not limited to exerting pulling forces, and applying optical tweezers to study pushing forces (specifically the force generated by a filament polymerizing into an obstruction) requires additional considerations.

Here we describe microengineered systems for broad study of polymerization forces and the role of compression and/or buckling in the behavior of biological filaments. This technology combines engineered micron-scale barriers to obstruct and restrain the tip of a filament, and optical tweezers to exert and measure the force at the tip. We demonstrate the utility of our method using microtubules (MTs) and a simple, single laser trap geometry to both exert force and measure growth rates. Our approach could be broadly adapted to study the important roles of polymer dynamics in cell biology including: lysteria locomotion, MT dynamics, actin, intermediate filaments, neurite outgrowth, acrosomal reactions and pseudopod and microspike extension (Lodish et al., 2000).

The approach reported here makes several important improvements over previous methods, elevating the study of polymerization forces to the accuracy and resolution that optical tweezers have brought to the study of motor proteins. We entirely eliminate reliance on analysis of image data and/or buckled filaments to determine the force on the MT tip or the growth rate (Dogterom and Yurke, 1997). Thus, our approach dramatically improves the resolution by eliminating sources of error associated with modeling the polymerizing MT as a buckling column and analysis of video microscopy data. Furthermore, the spatial and temporal resolution of back focal plane interferometry as employed in our system (Brouhard et al., 2003) greatly exceeds the light resolution limit and video data rate. Finally, we do not have to employ multiple traps and microbeads to control the MT (e.g., Kerssemakers et al., 2003), greatly simplifying experimental set up, instrument calibration and data collection.

\section{Design}

Such significant improvements were achieved by integrating a system of microfabricated structures with optical tweezers (Brouhard et al., 2003) and light microscopy. This integration required novel, engineered barriers with controlled three dimensional structure to allow full microscope and optical tweezers function while working with polymerizing filaments.

Figure 1 shows a side and top view of the structures. A sharp vertex in the top view constrains the MT tip, preventing thermal events from diverting the MT tip away from the intended obstruction. The angle, $\alpha$, is designed small enough to constrain the MT tip in the vertex but still large enough to avoid interference between the laser that forms

\footnotetext{
* Corresponding author.
} 


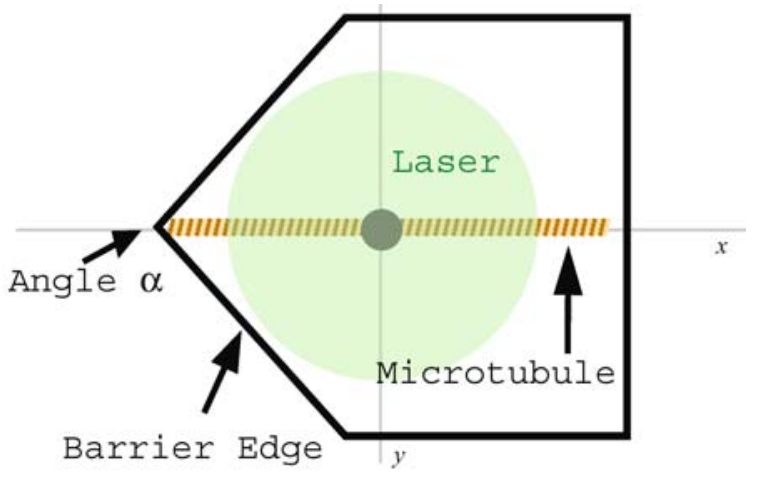

Top View

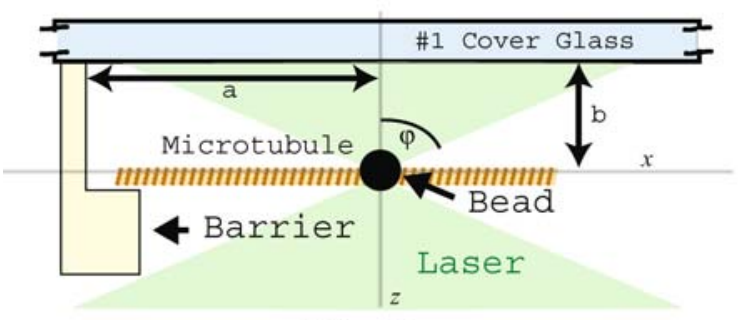

Side View
Fig. 1. Side and Top View Schematic of Experimental Configuration. In both views a trapped silica bead is shown attached to a MT polymerizing toward a barrier. Note objects are not drawn to scale; in particular actual beads are much larger relative to the MT. The top view shows the large laser foot print on the glass surface that results from the necessary tight focusing to achieve trapping. The angle $\alpha$ was carefully chosen to balance two important design requirements: $\alpha$ must be large enough to prevent the feature edges from interfering with the large laser diameter away from the focus but also small enough to reliably constrain the MT tip. A right angle is an effective compromise that avoids the interference that would result with smaller angles and right angles have proved to be adequate constraints (see results). The side view illustrates additional consideration for the barrier design. An undercut constrains the MT so that it can't slip away from the barrier in the z-axis. Avoiding laser interference is again an important consideration in designing the cross-sectional geometry of the barrier. For example, for $\varphi=67^{\circ}$ and assuming the laser focus is at the middle of the barrier height, $b=2 \mu \mathrm{m}$, a MT length $a=4.7 \mu \mathrm{m}$ is necessary to reach the barrier. Estimates of the buckling force for an MT of this length span 7-30 pN depending on how the attachment between bead and MT is modeled. The thinnest possible structure is desirable because it will allow experiments to begin with shorter MTs, increasing the length of time an individual MT can be studied and decreasing the likelihood of buckling. We have fabricated barriers ranging in height from 1.5-5 microns. As individual tubulin subunits squeeze onto the tip, the MT displaces the bead in trap, increasing the force at the tip.
A

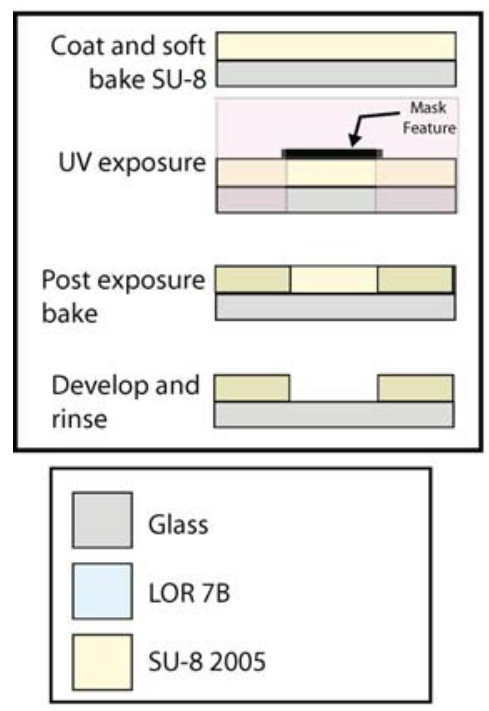

B

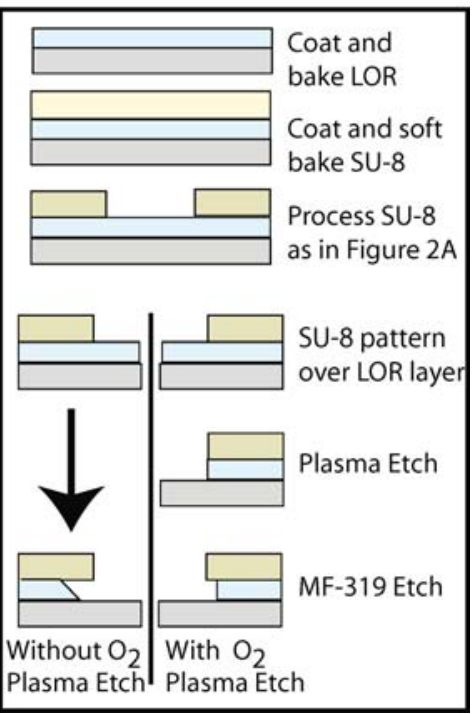

Fig. 2. Photoresist layer configurations and processing. (A) Single Layer Process. Dehydrated \#1 cover glass wafers (Eerie Scientific, Portsmouth, NH) are cleaned with the application of acetone and 2-propanol while spinning at 3000 RPM in a WS-400-6NPP-LITE coater (Laurell, North Wales, PA). After cleaning wafers are spin coated with SU-8 2002 or 2005 and soft baked. The soft bake begins at $65^{\circ} \mathrm{C}$ for 1 minute and concludes with 3 minutes at $95^{\circ} \mathrm{C}$. The coated wafer is exposed with $450 \mathrm{~mJ} / \mathrm{cm}^{2}$ of broad spectrum UV in a PLA-501FA aligner (Canon, Lake Success, NY). A post exposure bake (PEB) consisting of one minute at $65^{\circ} \mathrm{C}$, a ramp to $95^{\circ} \mathrm{C}$ and 1 minute at $95^{\circ} \mathrm{C}$ drives cross-linking of the SU-8 epoxy resin. Structures are developed for one minute in SU-8 Developer (Microchem, Newton, MA), rinsed for 30 seconds in 2-propanol and dried by spinning for 30 seconds at 3000 rpm. (B) Two-Layer Process. The two-layer process begins with the same dehydration and cleaning. LOR 3B or 7B (Microchem, Newton, MA) resist is spin coated at $3000 \mathrm{rpm}$. After coating, the LOR is baked for 20 minutes at $190^{\circ} \mathrm{C}$ to drive off solvent and set the etch rate. Following the LOR, $S U-8$ is coated and processed exactly as in the single layer process. With the SU-8 structure developed, the undercut can be created with two similar processes illustrated side by side in the figure. The left half shows the simplest process for creating undercut by etching for 30-500 seconds in MF-319 (Microchem, Newton, MA), and then rinsing in deionized water. The right half of the figure shows the process including an $\mathrm{O}_{2}$ plasma etch (100 $\mathrm{mT}$ $\mathrm{O}_{2}$, Power $=100 \mathrm{~W}, 1$ minute etch time) in an RIE 2000 SBT (South Bay Technology, San Clemente, CA) to remove the LOR directly beneath the primary SU-8 feature followed by an MF-319 to etch to create an undercut of depth less than one micron with a straight wall. 
the optical trap and the barriers. The side view shows the barrier's undercut sidewall profile which constrains the tip in the $z$-axis, preventing the tip from slipping down out of the structure and thereby avoiding the intended obstruction. The sidewall profile was created two different ways. The first relied on a negative tone photoresist to give slightly undercut sidewalls. This method could not provide the necessary control of the feature geometry so a second method was developed relying on a second layer of photoresist that could be etched out creating a true undercut.

The thickness of the photoresist layers forming the barriers is minimized to less than two microns. As shown in the side view of Figure 1, the low profile makes it possible to bring the laser focus within a few microns of the barrier and consequently to work with a short MT. Measurement of polymerization forces greater than 1-2 $\mathrm{pN}$ (Dogterom and Yurke, 1997; Schek and Hunt, 2003) requires a MT only a few microns long to avoid filament buckling prior to reaching the maximum polymerization force (Dogterom and Yurke, 1997; Gittes et al., 1996). Furthermore, the shortest possible MT at the beginning of the experiment maximizes the duration that polyermization under high loads can be observed before the MT is so long that it can no longer support the load without buckling.

The barrier structures are fabricated on \#1 cover glass substrate. Since the optical components of most microscopes are optimized for functioning with a \#1 microscope cover glass, this maintains the imaging abilities of the microscope and the trapping capabilities of the optical tweezers. Structures are sufficiently transparent to allow imaging and trapping through them during experiments. This results from constructing the structures from SU82002 or 2005 and LOR 3B or 7B (Microchem, Newton, MA) which are transparent and have a similar index of refraction to glass. Additionally, the structures are shelf stable for up to a month in a low humidity environment, function for several hours in the aqueous environment used for experiments and can be cleaned for repeated use. Process throughput is sufficient to make cleaning unnecessary, as each device can be treated as disposable if desired.

\section{Fabrication and Results}

Single-layer and two-layer processes were developed for creating an appropriate undercut profile. Figure 2 details processing and expected results of each method. All three methods utilize a $100 \mathrm{~mm}$, \#1 cover glass wafer (Eerie Scientific, Portsmouth, NH) as the substrate for the structures, and SU-8 photoresist for photolithography to establish primary structure patterns on the glass.
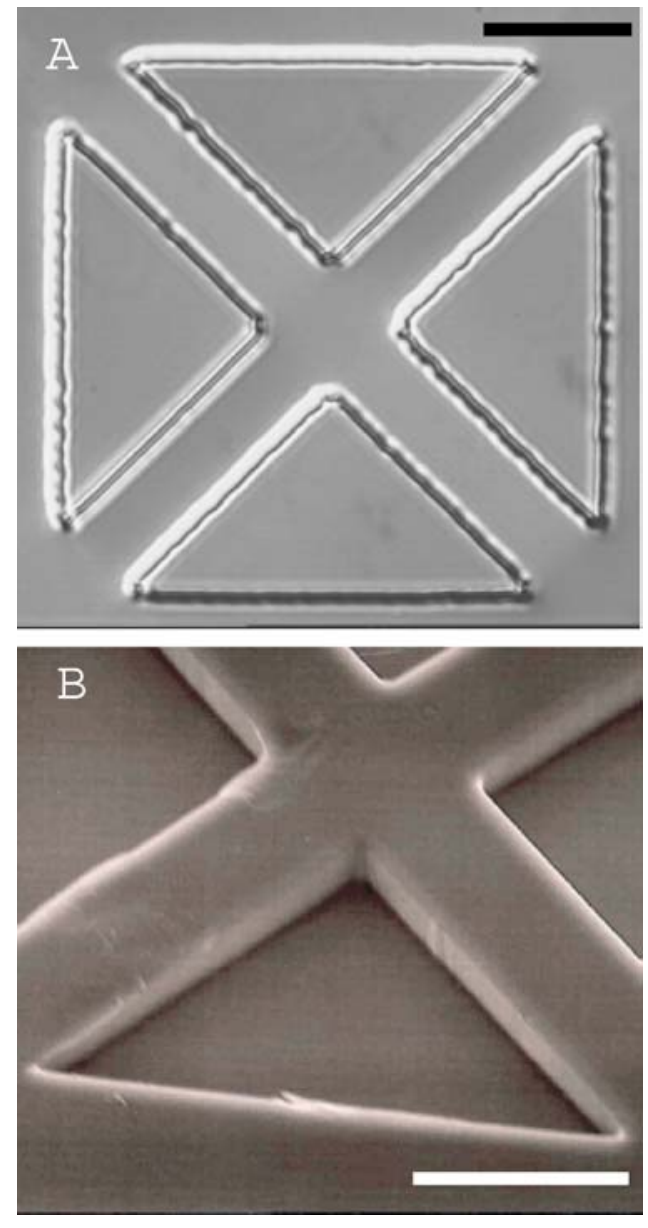

Fig. 3. Structures created with single-layer process. (A) Differential interference contrast (DIC) micrograph of a triangular pattern created using a single layer of SU-8 photoresist. (B) SEM of the same feature showing straight clean side walls and sharp vertices. While the features are sharp and clean they were unable to constrain MT tips sufficiently to result in efficient experiments. Scale bars are $14 \mu \mathrm{m}$.

In the case of the two-layer process, a layer of LOR below the SU-8 is used to facilitate fabrication of undercut structures.

The single layer process (Figure 2(A)) employs a single layer of epoxy based SU-8 2005 or 2002 photoresist directly on a cover glass wafer. SU-8 is a negative tone photoresist, which produces a vertical or slightly undercut sidewall profile when developed. Figure 3 shows a differential interference contrast (DIC) micrograph and scanning electron microscope image (SEM) of the structures produced with this single layer process. The SU-8 structures are sharp and clean and the SEM in Figure 3(B) shows nearly vertical sidewalls. The sidewall angle was not measured because it was apparent from MT experiments that this shallow undercut could constrain less than $5 \%$ of growing MT tips, and these were only temporarily 
impeded by the barrier before thermal events dislodged them from the undercut.

The low success rate using a single-layer structure lead to the development of a two-layer process providing better control of the undercut. This was accomplished by adding a layer of LOR 7B or 3B between the glass and SU-8 layer as shown in Figure 2(B). The LOR layer is isotropically etched with MF-319 (Microchem, Newton, MA) after the SU-8 structures are developed, resulting in a precisely controlled undercut. Since SU-8 is developed by an organic solvent and LOR is developed in aqueous solution the processes of creating the pattern and the undercut are decoupled.

The general two-layer processing procedure (Figure 2(B), left side) begins by spin-coating LOR onto the coverglas wafers, followed by a bake which establishes the LOR etch rate in subsequent steps. Next, the substrate is coated with SU-8 on top of the LOR layer, soft-baked, and exposed through a mask to define the primary two dimensional structure shown in the top view in Figure 1. A post-exposure bake crosslinks the exposed SU-8, and SU-8 developer (Microchem, Newton, MA) resolves the primary structures. Following development of the primary structures, the LOR is etched with MF-319 (Microchem, Newton, MA) to create the undercut profile. The final quality of the structures is most heavily dependent upon soft bake parameters and is only weakly determined by post exposure bake parameters. A 2-propanol rinse following SU-8 development is necessary to prevent the formation of an undeveloped layer of SU-8 in some features. Allowing the wafer to incubate at room temperature overnight prior to development greatly improves the repeatability of the process both from wafer to wafer and between locations on a single wafer.

Figure 4(A) shows a DIC image of a structure created with the two-layer process. The SU- 8 structures are sharp and the undercuts run closely parallel to the overlying SU-8 structures. Figure 4(B) shows the etch depth as a function of the etch time as measured from DIC micrographs of wafers processed in parallel. As expected, the relation is linear with small variations from the line likely created by uneven mixing during the etch or by inaccuracies associated with controlling short etch times. Once established, all aspects were repeatable with little variation except for the LOR etch rate, which showed as much as $500 \%$ variation day to day (data not shown), presumably due to changes in humidity, room temperature and the age of the MF-319 developer. However, etch rate was stable on any given day so that processing could easily be controlled after initial determination of the etch rate.

Figure 5 shows an image of a structure extensively etched to create a large undercut. A LOR pillar is seen supporting an SU-8 layer separated from the glass. The gap is remarkably uniform as shown by the fact that a
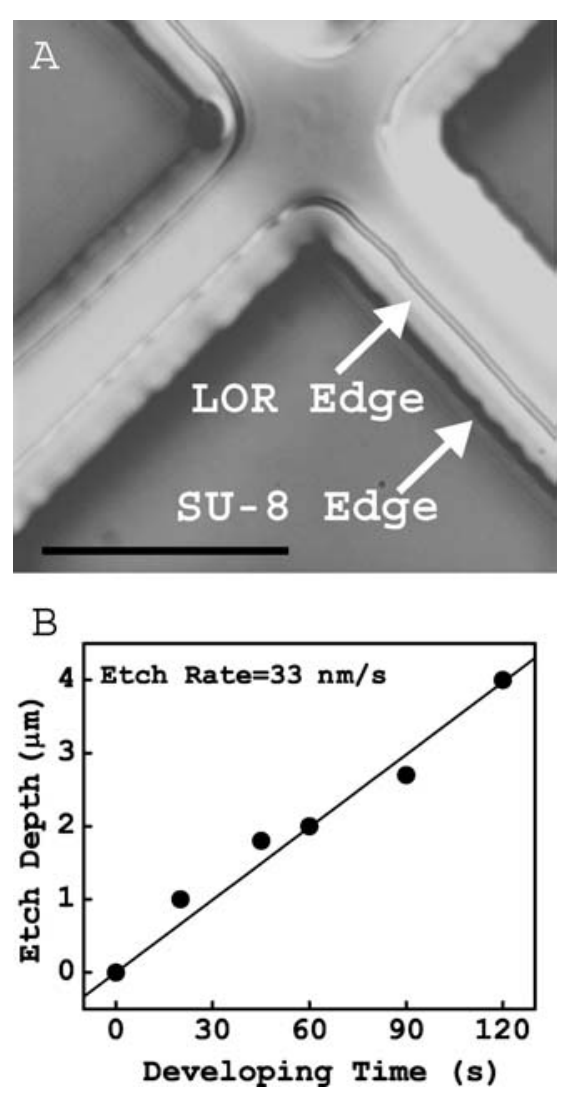

Fig. 4. Results from two-layer process. (A) DIC micrograph of a feature like the one shown in Figure 3, created with a two layer process resulting in an undercut. The scale bar is $14 \mu \mathrm{m}$. (B) Etch depth as a function of time. As expected the etch depth is linear with time.

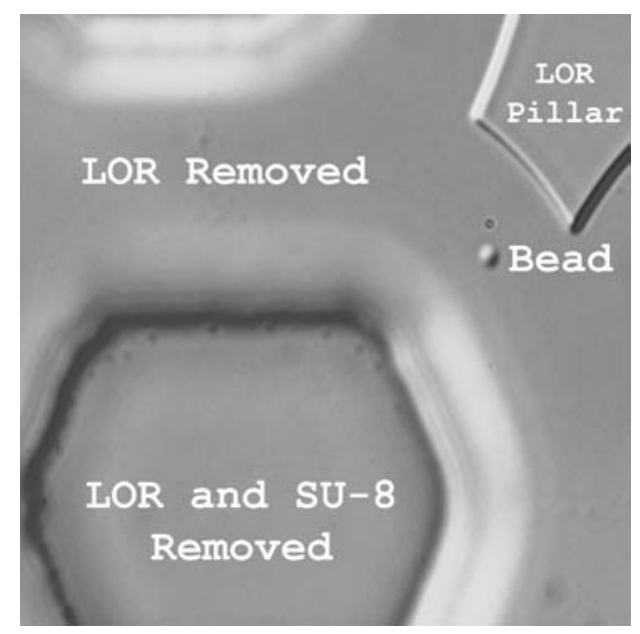

Fig. 5. Demonstration of large undercuts. DIC image of a very large undercut that results from an MF-319 etch of several minutes. The result is a large region surrounding the etched feature with a uniform gap between the glass substrate and the overlying SU-8 layer. A one micron diameter silica bead has been manipulated into the gap using optical tweezers. The gap height is only slightly larger than the bead diameter. The bead can be manipulated anywhere in the gap region but cannot be moved up or down, indicating that the gap is very uniform. 
one micron diameter silica bead (see figure) manipulated into the gap with optical tweezers (described in Brouhard et al., 2003) can be freely manipulated laterally, but is tightly constrained in the $z$-axis.

Creation of undercuts of a depth less than the thickness of the LOR layer was not possible because the etch time had to be long enough to etch away the entire layer of LOR below the primary SU-8 structure, resulting in
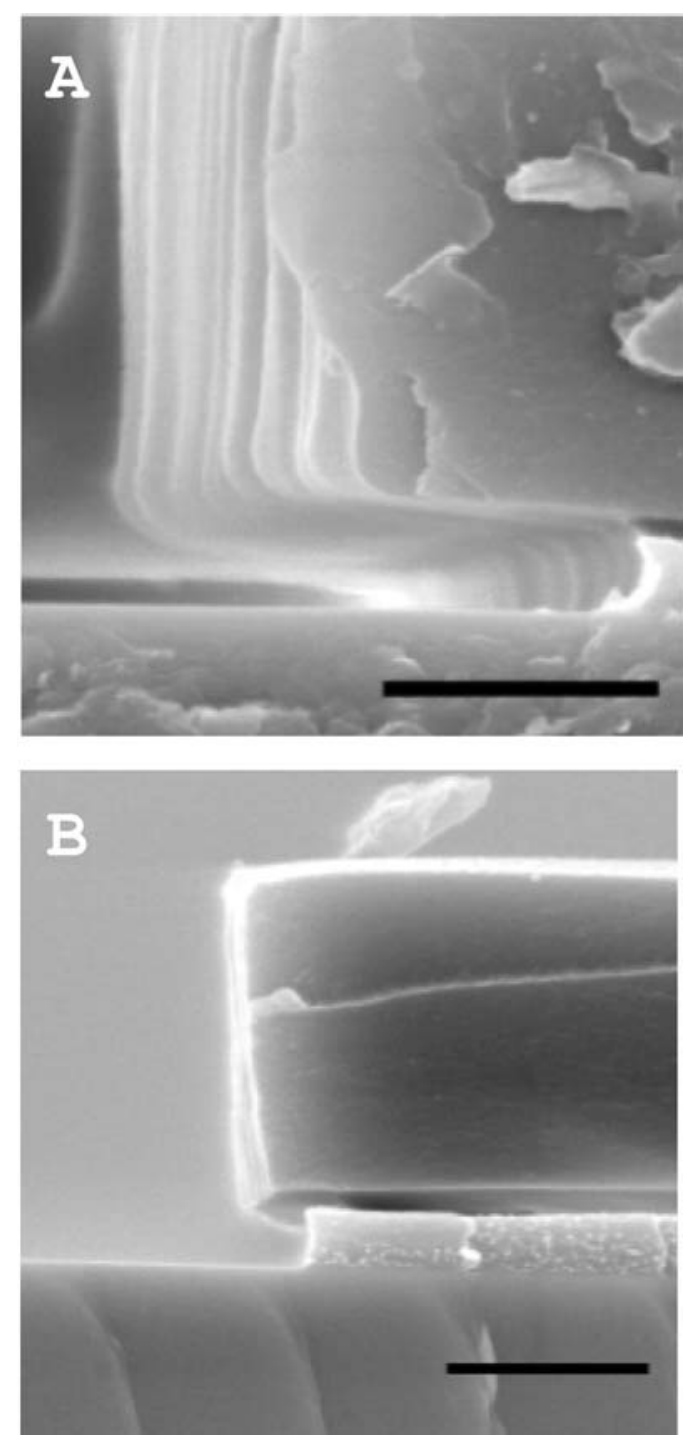

Fig. 6. Cross sectional scanning electron micrographs (SEMs). (A) Cross section of a feature manufactured without an oxygen plasma etch. The shape of the undercut is rounded and uniform. Undercuts must be at least as deep as the thickness of the LOR in order to remove the full layer of LOR. In this case an undercut of over $2 \mu \mathrm{m}$ was manufactured by etching longer than the minimum time necessary $(60 \mathrm{sec})$ to remove the LOR layer. (B) Cross section of a feature manufactured with an oxygen plasma etch to create a straight sidewall. The sidewall is very close to vertical and very shallow undercuts are possible such as $\sim 0.5 \mu \mathrm{m}$ shown here. Scale bars are $2 \mu \mathrm{m}$. an undercut at least as deep as the thickness of the LOR. To create a shallower undercut the two-layer process was modified with the addition of an oxygen plasma etch to remove the exposed LOR immediately below the areas where the SU-8 had been removed in creation of the primary features (Figure 2(B), right side). An additional aspect of the plasma etch is that the undercut terminates with a straight sidewall rather than angled or rounded sidewall. In Figure 6(A) a cross-section SEM of the undercut features created without the oxygen plasma etch shows the rounded sidewall of the etched area and an undercut depth exceeding the thickness of the LOR layer. In Figure 6(B), the $\mathrm{O}_{2}$ etch was used to produce an undercut that is very narrow $(\sim 0.5 \mu \mathrm{m})$ with a straight sidewall.

Figure 7 shows a video enhanced DIC micrograph of an experiment in which the tip of an MT is subjected to a controlled load. A MT-bound bead is held in the laser trap while the polymerizing MT grows into the barrier. Images of the MT in a straight unloaded configuration, and at a later time loaded and bent, are superimposed (see the figure caption for details). Tips that encounter the barrier produced using the two-layer process are blocked and constrained in greater than $90 \%$ of encounters making experiments much more efficient than with the single layer process.

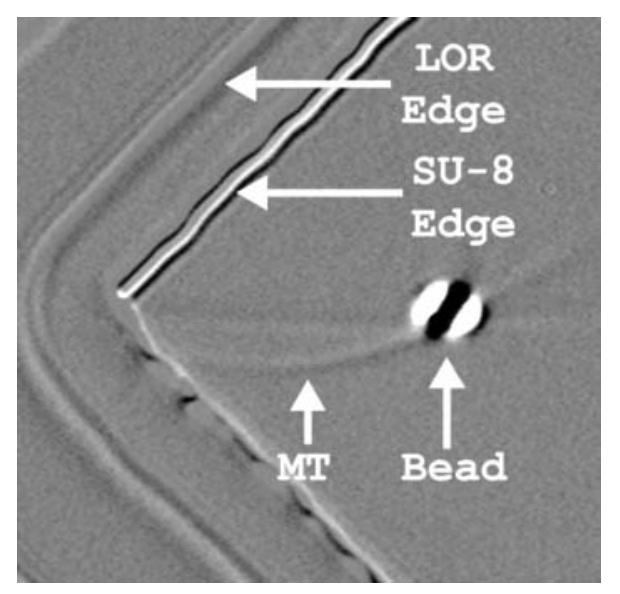

Fig. 7. DIC micrograph of an experiment to study MT polymerization forces. The bead is shown at the center of a triangular feature with the MT extending from the bead to the barrier. In this image, a background image is subtracted from the later image (in addition to contrast enhancement) making the MT visible but also retaining the earlier image of the MT which can be seen as a faint line extending straight from the bead to the vertex of the barrier. When the MT initially encounters the barrier it is unloaded and straight. Over time polymerization pushes the bead out of the trap until the restoring force is sufficient to cause the MT to buckle. In this case a MT long enough to buckle was chosen to provide visual evidence of the force at the tip. A typical experiment ends in this configuration with the MT buckled as it has grown to a length that no longer supports the loads developed by polymerization. 


\section{Discussion}

We have developed a process that produces undercut structures to reliably constrain and study microfilament mechanics, as shown in Figure 7. These heavily crosslinked SU-8 structures remain sharp and stable for weeks when stored in a low humidity enivronment, and are compatible with aqueous environments for several hours. The structures are sufficiently robust to withstand repeated use, provided the structures are thoroughly dehydrated after cleaning.

Most importantly the structures meet the necessary design requirements for use in conjunction with high quality light imaging and optical tweezers. They are produced on high optical quality cover glass, are in all locations sufficiently transparent to allow imaging during an experiment, avoid laser interference when the bead is maneuvered close to the barrier, and maintain excellent image quality as shown in Figure 7. Additionally, the manufacturing process is very flexible; undercut depth is easily tuned from as little as $300 \mathrm{~nm}$ to as much as tens of microns, and the thickness of the two layer structures can be reduced below $1.5 \mu \mathrm{m}$ with no changes in the process, other than going to higher spin coating speeds. LOR layers less than $500 \mathrm{~nm}$ and SU-8 layers of $\sim 1$ micron are easily attainable with no modification of commercially available products. The decoupling of the processing of the primary structure in SU-8 from processing for the undercut in LOR, allow these aspects to be varied independently. Finally, processing throughput easily meets the needs of biological experiments.

These considerations are not unique to experiments studying polymer mechanics, and may apply to any system designed for use with laser trapping and light imaging. As micro and nanotechnology increasingly incorporate aspects from biology, it is necessary to develop these devices along lines that will allow visualization and manipulation under a light microscope (e.g. Baldi et al., 2003; Brown and Hancock, 2002; Hoff et al., 2004; Jia et al., 2004). Additionally, it is not difficult to imagine scenarios requiring optical tweezers to carefully assemble and characterize devices.

This technology is a critical step for studying the dynamics of polymerization based motility, enabling the study of polymerization "motors" at the same resolution and efficiency that has yielded much valuable information about classic motor proteins such as kinesin and myosin. In the case of MTs, we are applying this methodology to study dynamic instability and how MT associated proteins modify MT polymerization dynamics.

\section{Acknowledgments}

The authors would like to thank Edgar Meyhofer, ChihTing Lin and Katsuo Kurabayashi for valuable help learning lithography techniques and Jeremy Hoff, Elissa Burk, David Lorch and E.F. Hasselbrink for comments on the manuscript. Additionally, the chemical engineering clean room at the University of Michigan supplied equipment and expertise. Kevin Ke assisted with the SEMs. This work was supported by the Burroughs Wellcome Fund and NSF. Henry Schek is supported by the Whitaker Foundation.

\section{References}

M.W. Allersma, F. Gittes, M.J. deCastro, R.J. Stewart, and C.F. Schmidt, Two-Dimensional Tracking of ncd Motility by Back Focal Plane Interferometry 74, 1074 (1998).

A. Baldi, J.N. Fass, M.N. De Silva, D.J. Odde, and B. Ziaie, A MicroTool for Mechanical Manipulation of in vitro Cell Arrays 5, 291 (2003).

G.J. Brouhard, H.T. Schek, and A.J. Hunt, Advanced Optical Tweezers for the Study of Cellular and Molecular Biomechanics 50, 121 (2003).

T.B. Brown and W.O. Hancock, A Polarized Microtubule Array for Kinesin-Powered-Nanoscale Assembly and Force Generation 2, 1131 (2002).

M. Dogterom and B. Yurke, Measurement of the Force-Velocity Relation for Growing Microtubules 278, 856 (1997).

H. Felgner, R. Frank, and M. Schliwa, Flexural Rigidity of Microtubules Measured with the Use of Optical Tweezers 109, 509 (1996).

F. Gittes, E. Meyhofer, S. Baek, and J. Howard, Directional Loading of the Kinesin Motor Molecule as it Buckles a Microtubule 70, 418 (1996).

J.D. Hoff, L.J. Cheng, E. Meyhofer, L.J. Guo, and A.J. Hunt, Nanoscale Protein Patterning by Imprint Lithography 4, 853 (2004).

L.L. Jia, S.G. Moorjani, T.N. Jackson, and W.O. Hancock, Microscale Transport and Sorting by Kinesin Molecular Motors 6, 67 (2004).

J.W.J. Kerssemakers, M.E. Janson, A. van der Horst, and M. Dogterom, Optical Trap Setup for Measuring Microtubule Pushing Forces 83, 4441 (2003).

H. Lodish, A. Berk, S. Zipursky, P. Matsudaira, D. Baltimore, and J. Darnell, Molecular Cell Biology (W.H. Freeman and Company, New York, NY, 2000) pp. 796-843.

A.D. Mehta, K.A. Pullen, and J.A. Spudich, Single Molecule Biochemistry Using Optical Tweezers 430, 23 (1998).

J.C. Meiners and S.R. Quake, Femtonewton Force Spectroscopy of Single Extended DNA Molecules 84, 5014 (2000).

H.T. Schek and A. Hunt, Studying the Force Generated by Microtubule Polymerization with Optical Tweezers 84, 113A (2003).

K. Visscher, M.J. Schnitzer, and S.M. Block, Single Kinesin Molecules Studied with a Molecular Force Clamp 400, 184 (1999). 